\title{
ON THE UNRAMIFIED BRAUER GROUP OF A HOMOGENEOUS SPACE
}

\author{
MIKHAIL BOROVOI
}

\begin{abstract}
We give a new proof of the theorem stating that for any connected linear algebraic group $G$ over an algebraically closed field $k$ of characteristic 0 and for any connected closed subgroup $H$ of $G$, the unramified Brauer group of $G / H$ vanishes.
\end{abstract}

\section{INTRODUCTION}

In this note $k$ always denotes an algebraically closed field of characteristic 0 . For an irreducible algebraic variety $X$ over $k$, we denote by $k(X)$ the field of rational functions on $X$. We denote by $\mathrm{Br}_{\mathrm{nr}} k(X)$, or just by $\mathrm{Br}_{\mathrm{nr}} X$, the unramified Brauer group of $k(X)$ with respect to $k$, see [CTS, Def. 5.3].

We give a new proof of the following theorem:

Theorem 1 ([[BDH, Thm. 5.1]). Let $G$ be a connected linear algebraic group over an algebraically closed field $k$ of characteristic 0 , and let $H \subset G$ be a connected closed subgroup. Then $\mathrm{Br}_{\mathrm{nr}} k(G / H)=0$.

In the case when $G$ is simply connected this is a classical result of Bogomolov [Bog, Thm. 2.4], see Colliot-Thélène and Sansuc [CTS, Thm. 9.13]. Bogomolov proved his theorem by a topological method in the case $k=\mathbb{C}$, but the general case of an algebraically closed field $k$ of characteristic 0 reduces to the case $k=\mathbb{C}$ by the Lefschetz principle, see [CTS], beginning of $\S 9$. Our Theorem 1 answers affirmatively a question of Colliot-Thélène and Sansuc in [CTS, Rem. 9.14] and a question after Theorem 1.4 in the paper $[$ [TK $]$ by Colliot-Thélène and Kunyavski1̌. Theorem 1 was recently proved in the preprint $[\overline{\mathrm{BDH}}]$ of Demarche, Harari and the author by a numbertheoretical method. Here we deduce this theorem from Bogomolov's theorem.

Acknowledgements. The author is very grateful to Cyril Demarche for his helpful suggestions, which permitted to considerably shorten and simplify the proof. The author thanks Jean-Louis Colliot-Thélène for useful remarks.

2000 Mathematics Subject Classification. Primary: 14F22 ; Secondary 14M17, 14L10. Key words and phrases. Unramified Brauer group, homogeneous space, linear algebraic group.

Partially supported by the Hermann Minkowski Center for Geometry. 


\section{NOTATION AND PRELIMINARIES}

By $k$ we always denote an algebraically closed field of characteristic 0 . Let $G$ be a connected linear algebraic group over $k$. We use the following notation:

$G^{\mathrm{u}}$ is the unipotent radical of $G$;

$G^{\mathrm{red}}=G / G^{\mathrm{u}}$, it is reductive;

$G^{\mathrm{ss}}=\left[G^{\mathrm{red}}, G^{\mathrm{red}}\right]$, it is semisimple;

$G^{\text {tor }}=G^{\text {red }} / G^{\text {ss }}$, it is a torus;

$G^{\mathrm{ssu}}=\operatorname{ker}\left[G \rightarrow G^{\mathrm{tor}}\right]$, it is an extension of a semisimple group $G^{\mathrm{ss}}$ by a unipotent group $G^{\mathrm{u}}$.

Note that $G^{\text {tor }}$ is the largest quotient torus of $G$. Note also that Pic $G=0$ if and only if $G^{\text {ssu }}$ is simply connected, cf. [Sa], Lemma 6.9 and Remark 6.13 .

Let $X$ be a smooth integral variety over $k$. If $V$ is a smooth compactification of $X$ (existing by Hironaka's theorem), then we can identify $\mathrm{Br}_{\mathrm{nr}} X$ with $\operatorname{Br} V$, cf. [CTS, Thm. 5.11(iii)]. We regard $\mathrm{Br}_{\mathrm{nr}} X=\mathrm{Br} V$ as a subgroup of $\operatorname{Br} X$, cf. [CTS, Thm. 5.11(ii)]. If $f: X_{1} \rightarrow X_{2}$ is a morphism of smooth integral varieties defined over $k$, one can extend $f$ to a morphism of suitable smooth compactifications $f^{\prime}: V_{1} \rightarrow V_{2}$, where $V_{i}$ is a smooth compactification of $X_{i}(i=1,2)$, see [BK, $\left.\S 1.2 .2\right]$ (again, one uses Hironaka's theorem). It follows that $f$ induces a homomorphism of the unramified Brauer groups $f^{\mathrm{nr}}: \mathrm{Br}_{\mathrm{nr}} X_{2} \rightarrow \mathrm{Br}_{\mathrm{nr}} X_{1}$ fitting into a commutative diagram

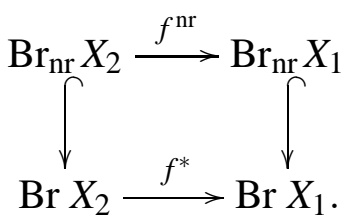

\section{Reduction to a SPeCial CASE}

Let $G$ be a connected linear algebraic group defined over $k$, and let $H \subset G$ be a connected closed subgroup.

3.1. Reduction to the case Pic $G=0$. It is well known (see e.g. [Bor, Lemma 5.2]) that there exists a connected linear algebraic group $G^{\prime}$ over $k$ with Pic $G^{\prime}=0$ and a connected closed subgroup $H^{\prime} \subset G^{\prime}$, such that the varieties $G / H$ and $G^{\prime} / H^{\prime}$ are isomorphic. Therefore, we may and shall assume that $G$ in Theorem 1 satisfies Pic $G=0$.

3.2. Reduction to the case $H=H^{\text {ssu }}$. Consider the subgroup $H^{\text {ssu }} \subset H$. The map $G / H^{\text {ssu }} \rightarrow G / H$ is a right $H^{\text {tor }}$-torsor. Since $H^{\text {tor }}$ is a split torus, by Hilbert's Theorem 90 this torsor admits a local section. Thus the homogeneous space $G / H^{\mathrm{ssu}}$ is birationally equivalent to $G / H \times{ }_{k} H^{\text {tor }}$, and by [CTS, Prop. 5.7] we have $\operatorname{Br}_{\mathrm{nr}}\left(G / H^{\text {ssu }}\right) \cong \mathrm{Br}_{\mathrm{nr}}(G / H)$. Therefore, we may and shall assume in Theorem 1 that $H=H^{\mathrm{ssu}}$, i.e. $H$ is character-free. 


\section{Deduction of TheOREM 1 from Bogomolov's THEOREM}

Consider the map $G \rightarrow G / H$. Since the variety of $G$ is rational, we have $\mathrm{Br}_{\mathrm{nr}} G=0$, and we see from diagram (1) that

$$
\mathrm{Br}_{\mathrm{nr}}(G / H) \subset \operatorname{ker}[\mathrm{Br}(G / H) \rightarrow \mathrm{Br} G] .
$$

Taking in account the results of Section 3, we now assume that Pic $G=0$ and that $H \subset G$ is connected and character-free. Set $G_{1}=G^{\text {ssu }}$, then $G_{1}$ is simply connected because Pic $G=0$, see $\S 2$. Since $H$ is character-free, we have $H \subset G_{1}$.

Let $i: G_{1} \hookrightarrow G$ denote the inclusion homomorphism. Consider the following commutative diagram of morphisms of varieties:

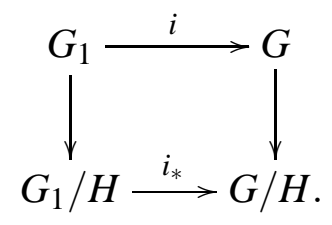

By functoriality (see $\S 2$ ) this diagram defines a homomorphism $i^{\mathrm{nr}}: \mathrm{Br}_{\mathrm{nr}}(G / H) \rightarrow$ $\operatorname{Br}_{\mathrm{nr}}\left(G_{1} / H\right)$ fitting into a commutative diagram

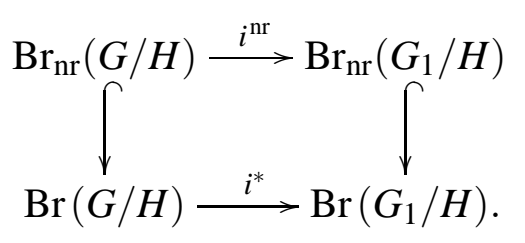

Note that the map $i: G_{1} \rightarrow G$ in diagram (3) is an $H$-equivariant map from the right $H$-torsor $G_{1}$ over $G_{1} / H$ to the right $H$-torsor $G$ over $G / H$. Sansuc's exact sequence [Sa, (6.10.1)], applied to this diagram, gives a commutative diagram with exact rows

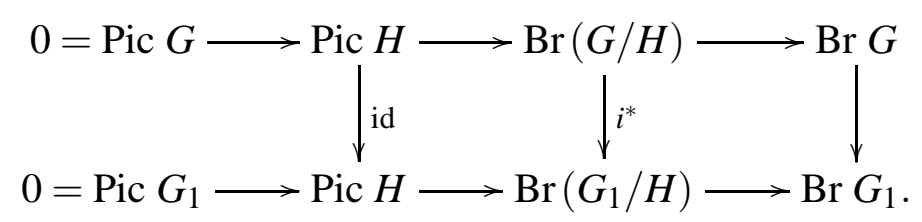

We see from (5) that the homomorphism $i^{*}$ restricted to $\operatorname{ker}[\operatorname{Br}(G / H) \rightarrow$ $\mathrm{Br} G]$ is injective, and we see from (2) that $i^{*}$ restricted to $\operatorname{Br}_{\mathrm{nr}}(G / H)$ is injective. Now it follows from diagram (4) that the homomorphism

$$
i^{\mathrm{nr}}: \mathrm{Br}_{\mathrm{nr}}(G / H) \rightarrow \mathrm{Br}_{\mathrm{nr}}\left(G_{1} / H\right)
$$

is injective. Since $G_{1}$ is simply connected and $H$ is connected, by Bogomolov's theorem [CTS, Thm. 9.13] we have $\operatorname{Br}_{\mathrm{nr}}\left(G_{1} / H\right)=0$. We conclude that $\mathrm{Br}_{\mathrm{nr}}(G / H)=0$, which proves Theorem 1 . 


\section{REFERENCES}

[Bog] F. A. Bogomolov, Brauer groups of the fields of invariants of algebraic groups (Russian), Mat. Sb. 180 (1989), 279-293; English transl.: Math. USSR Sb. 66 (1990), 285-299.

[Bor] M. Borovoi, The Brauer-Manin obstruction to the Hasse principle for homogeneous spaces with connected or abelian stabilizer, J. reine angew. Math. $\mathbf{4 7 3}$ (1996), 181-194.

[BDH] M. Borovoi, C. Demarche et D. Harari, Complexes de groupes de type multiplicatif et groupe de Brauer non ramifié des espaces homogènes, arXiv: 1203.5964v1 [math.AG].

[BK] M. Borovoi and B. È. Kunyavskiı̆, Formulas for the unramified Brauer group of a principal homogeneous space of a linear algebraic group, J. Algebra 225 (2000), 804-821.

[CTK] J.-L. Colliot-Thélène et B. È. Kunyavskiĭ, Groupe de Picard et groupe de Brauer des compactifications lisses d'espaces homogènes, J. Algebraic Geom. 15 (2006), $733-752$.

[CTS] J.-L. Colliot-Thélène and J.-J. Sansuc, The rationality problem for fields of invariants under linear algebraic groups (with special regards to the rationality problem), in: Algebraic groups and homogeneous spaces, pp. 113-186, Tata Inst. Fund. Res. Stud. Math., Tata Inst. Fund. Res., Mumbai, 2007.

[Sa] J.-J. Sansuc, Groupe de Brauer et arithmétique des groupes algébriques linéaires sur un corps de nombres, J. reine angew. Math. 327 (1981), 12-80.

Raymond and BeVerly SaCKLer School of Mathematical Sciences, Tel AVIV UNIVERSITy, 69978 Tel AVIV, ISRAEL

E-mail address: borovoi@post.tau.ac.il 